\title{
Author Correction: Spectroscopic fingerprint of charge order melting driven by quantum fluctuations in a cuprate
}

\author{
W. S. Lee (iD), Ke-Jin Zhou (D), M. Hepting (iD, J. Li, A. Nag, A. C. Walters, M. Garcia-Fernandez, H. C. Robarts,
} M. Hashimoto, H. Lu, B. Nosarzewski, D. Song, H. Eisaki, Z. X. Shen (D), B. Moritz $\mathbb{D}$, J. Zaanen and T. P. Devereaux $\mathbb{D}$

Correction to: Nature Physics https://doi.org/10.1038/s41567-020-0993-7, published online 31 August 2020.

In the version of this Letter originally published, the name of the author Ke-Jin Zhou was incorrectly given as 'Ke-Jin J. Zhou'. It has now been amended in all versions of the Letter.

Published online: 9 October 2020

https://oi.org/10.1038/s41567-020-01075-1

๑ The Author(s), under exclusive licence to Springer Nature Limited 2020

\section{Publisher Correction: Nanoscale mechanics of antiferromagnetic domain walls}

Natascha Hedrich (D), Kai Wagner (D), Oleksandr V. Pylypovskyi (D), Brendan J. Shields (D), Tobias Kosub, Denis D. Sheka (D), Denys Makarov (D) and Patrick Maletinsky (D)

Correction to: Nature Physics https://doi.org/10.1038/s41567-020-01157-0, published online 15 February 2021.

In the PDF version of this Letter originally published online, all instances of ' $\boldsymbol{\mu}$ ' were missing in equations (5) and (6); the PDF has now been updated. The HTML version was correct.

Published online: 2 March 2021

https://doi.org/10.1038/s41567-021-01205-3

(c) The Author(s), under exclusive licence to Springer Nature Limited 2021

\section{Author Correction: Topological limits to the parallel processing capability of network architectures}

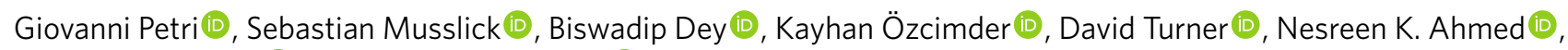
Theodore L. Willke (D) and Jonathan D. Cohen (D)

Correction to: Nature Physics https://doi.org/10.1038/s41567-021-01170-x, published online 18 February 2021.

In the version of this Article originally published online, the first name of the author Theodore L. Willke was spelt incorrectly as 'Theodeore'. Furthermore, the text "S.M. and J.D.C. acknowledge support from the John Templeton Foundation. The opinions expressed in this publication are those of the authors and do not necessarily reflect the views of the John Templeton Foundation." was missing from the Acknowledgements. All versions of the Article have been amended.

Published online: 5 March 2021

https://doi.org/10.1038/s41567-021-01212-4

(c) The Author(s), under exclusive licence to Springer Nature Limited 2021 